\title{
Estudio cuasi experimental sobre la efectividad de la musicoterapia para reducir la ansiedad ante una técnica intervencionista en la unidad del dolor
} Quasi-experimental study on effectiveness of music therapy to reduce anxiety before an interventional technique in the pain unit

M. M. Monerris , M. J. Medina Gómez², A. Caparrós Giménez², M. Aguas Compaired ${ }^{3}$ y M. J. Simón Solano¹

${ }^{1}$ Servicio de Anestesiología, Reanimación y Terapéutica del Dolor. Hospital Universitario Sagrat Cor de Barcelona, España. ${ }^{2}$ Departamento de Enfermería Quirúrgica. Hospital Universitario Sagrat Cor de Barcelona, España. ${ }^{3}$ Servicio de Farmacia Hospitalaria. Hospital Universitario Sagrat Cor de Barcelona, España

\section{ABSTRACT}

Objective: To assess the efficacy of music therapy in reducing anxiety in patients undergoing interventional technique (IT) in the Pain Unit (UD) of the University Hospital Sagrat Cor in Barcelona.

Material and method: Quasi-experimental study with a control group, prospective, pre- and post-intervention, transversal, non-randomized. Approved by the Research Ethics Committee IDC Salud Catalunya. Study population: patients scheduled to perform IT in the UD. Inclusion criteria: > 18 years old. IT indication. Exclusion criteria: psychiatric disorder, inability to answer study variables. Intervention group: IT has been performed by randomly playing the chosen music, free of noise pollution. Control group: IT has been performed without music therapy.

The modified analog visual scale has been used as a measuring instrument to assess the level of anxiety.

Results: 80 patients $(71.6 \%$ women, mean age 66.7 (SD 14) years, $49.4 \%$ average studies, $64.2 \%$ pensioners). $60.5 \%$ do not perform treatment with anxiolytics. $59 \%$ have low back pain, caudal epidural block is performed. $33.3 \%$ underwent interventional technique for the first time.

In the control group the value of intra VAS is 5.83 (SD 3.2) and in the music therapy group 5.0 (SD 2.2), this difference is not statistically significant $(F=1.614$, $p=0.208$ ). The same happens in the value of post-intervention EVA.

\section{RESUMEN}

Objetivos: Valorar la eficacia de la musicoterapia en la disminución de la ansiedad en pacientes a los que se les realiza técnica intervencionista (TI) en la Unidad del Dolor (UD) del Hospital Universitario Sagrat Cor de Barcelona.

Material y método: Estudio cuasi experimental con grupo control, prospectivo, pre y postintervención, transversal, no aleatorizado. Aprobado por el Comité Ético de Investigación IDC Salud Catalunya. Población estudio: pacientes programados para realización de TI en la UD. Criterios inclusión: > 18 años de edad. Indicación de TI. Criterios exclusión: trastorno psiquiátrico, incapacidad contestar variables de estudio. Grupo intervención: se ha realizado la TI reproduciéndose aleatoriamente la música elegida, libre de contaminación acústica. Grupo control: se ha realizado la $\mathrm{TI}$ sin musicoterapia.

Se ha empleado la escala visual análoga modificada como instrumento de medida para valorar el nivel de ansiedad.

Resultados: 80 pacientes $[71,6 \%$ mujeres, media de edad 66,7 [SD 14] años, 49,4 \% estudios medios, $64,2 \%$ pensionistas). El 60,5\% no realiza tratamiento con ansiolíticos. El $59 \%$ presenta lumborradiculalgia, se les realiza bloqueo epidural caudal. Al 33,3\% se les realiza técnica intervencionista por primera vez.

En el grupo control el valor de EVA intra es de 5,83 (SD 3,2) y en el grupo de musicoterapia de 5,0 (SD 2,2), esta diferencia no es estadísticamente significativa $(F=$

Recibido: 22-09-2019

Aceptado: 13-01-2020

Correspondencia: María del Mar Monerris mmmonerris.germanstrias@gencat.cat
Monerris MM, Medina Gómez MJ, Caparrós Giménez A, Aguas Compaired M, Simón Solano MJ. Estudio cuasi experimental sobre la efectividad de la musicoterapia para reducir la ansiedad ante una técnica intervencionista en la unidad del dolor. Rev Soc Esp Dolor 2020;27(1):7-15 
In the control group the value of post VAS is 3.7 (SD 3.3) and in the music therapy group 3.1 (SD 2.4), this difference is not statistically significant $(F=0.755, p=$ 0.387).

In the control group the value of the intra analog visual scale is 4.3 (SD 3.1) and in the music therapy group 3.0 (SD 2.0), this difference is statistically significant $(F=4$, $83 \mathrm{p}=0.031$ ).

In the control group the value of the post analog visual scale is 2.7 (SD 2.8) and in the music therapy group 1.3 (SD 1.5), this difference is also statistically significant ( $F$ $=7.427, p=0.008$ )

$81.5 \%$ consider that they have received enough information about the interventionist technique and $18.5 \%$ are satisfied.

95.1\% consider that the professionals have given him confidence and security and the remaining $4.9 \%$ are considered satisfied.

Of those who have assessed (40 patients) if music has created a relaxed atmosphere, $80 \%$ are considered very satisfied and the remaining $20 \%$ satisfied.

Conclusion: Although pain is not significantly improved in the interventionist group, anxiety does in both the intra and post-IT phases.

Patients feel very satisfied about the information received and consider the confidence and security provided by professionals very satisfactory. In addition, the music therapy group considers, in general, the relaxed atmosphere created by the music.

Music therapy is an excellent therapeutic tool, easy to use, accessible and economical, which can be used as an adjunct in IT in the UD.

Key words: Pain, anxiety, music therapy, interventional techniques.
1,614, $p=0,208$ ). En el valor de EVA postintervención sucede lo mismo.

En el grupo control el valor de EVA post es de 3,7 (SD 3,3 ) y en el grupo de musicoterapia de 3,1 (SD 2,4); esta diferencia no es estadísticamente significativa $(F=0,755$, $p=0,387)$.

En el grupo control el valor de la escala visual analógica intra es de 4,3 (SD 3,1) y en el grupo de musicoterapia de 3,0 (SD 2,0), esta diferencia sí es estadísticamente significativa ( $F=4,83, p=0,031$ ).

En el grupo control el valor de la escala visual analógica post es de 2,7 (SD 2,8) y en el grupo de musicoterapia de 1,3 (SD 1,5); esta diferencia también es estadísticamente significativa $(F=7,427, p=0,008)$.

El $81,5 \%$ considera que ha recibido suficiente información sobre la técnica intervencionista y el 18,5 \% están satisfechos.

El 95,1\% considera que los profesionales le han aportado confianza y seguridad y el $4,9 \%$ restante se consideran satisfechos.

De los que han valorado (40 pacientes) si la música ha creado un ambiente relajado el $80 \%$ se consideran muy satisfechos y el $20 \%$ restante satisfechos.

Conclusión: Aunque el dolor no se ve mejorado significativamente en el grupo intervencionista sí lo hace la ansiedad tanto en la fase intra como en la post Tl.

Los pacientes se sienten muy satisfechos sobre la información recibida y consideran muy satisfactoria la confianza y seguridad que les aportan los profesionales. Además, el grupo de musicoterapia considera, en general, muy satisfactorio el ambiente relajado que les crea la música.

La musicoterapia es una excelente herramienta terapéutica, fácil de usar, accesible y económica, que puede utilizarse como coadyuvante en las $\mathrm{TI}$ en la UD.

Palabras clave: Dolor, ansiedad, musicoterapia, técnicas intervencionistas.

\section{INTRODUCCIÓN}

La ansiedad es una sensación anormal, abrumadora, de aprensión y miedo, que produce cambios fisiológicos como diaforesis, hipertensión y taquicardia. Se produce por dudas respecto a la realidad y naturaleza de la amenaza o por inseguridad de la propia capacidad para hacer frente a ella $[1,2]$.

Actualmente existe un creciente interés por aliviar la ansiedad de los pacientes (3) en cualquier situación que se desarrolle en el ambiente hospitalario.

La intervención musical es un recurso terapéutico que está siendo cada vez más utilizado como terapia complementaria, para promover relajación, confort emocional y espiritual, distracción, sensación de bienestar y alivio del dolor [4].

La música siempre ha estado vinculada a la vida social del hombre, cumpliendo diferentes funciones sociales [5]. Una de estas funciones es la aplicación de la música en la medicina. En un contexto hospitalario, la musicoterapia se ha mostrado eficaz, principalmente como forma de reducir la ansiedad [6].

Al hablar de musicoterapia nos estamos refiriendo a cómo puede ser utilizada la música de manera estudiada y controlada para modular las respuestas de las personas que la escuchan. Estos efectos pueden ser estimulantes y sedantes, ansiolíticos, analgésicos, etc. (7).

Ante una situación de ansiedad la respiración suele verse bloqueada. La música puede actuar como guía para acompasar y controlar la respiración y ayudar a focalizar la atención en el ejercicio de relajación [7], mejorando la motivación y los sentimientos positivos de autocontrol frente al dolor (8).

Numerosos estudios han demostrado la interacción entre ansiedad y dolor. La ansiedad es la forma más común de deterioro del bienestar subjetivo en pacientes con dolor crónico, destacando la ansiedad situacional causada por situaciones como procedimientos invasivos [9]. 
Los pacientes que acuden a la Unidad del Dolor frecuentemente presentan estrés y ansiedad ante la realización de una técnica intervencionista.

Un ambiente tranquilo y confortable, sin contaminación acústica, junto con una colocación adecuada del paciente en la camilla de procedimientos, son puntos clave de la asistencia durante la realización de una técnica intervencionista. Precisa de conocimientos del personal sanitario, así como estar familiarizado con la técnica intervencionista programada. Se deberá también tener en cuenta la técnica intervencionista a realizar, los fármacos a administrar, edad, talla, peso, antecedentes del paciente y las medidas de seguridad (posición del cuerpo, el perfecto funcionamiento de la camilla de procedimientos, las normas de protección del paciente y la previsión del equipo necesario].

\section{Justificación del estudio}

Bringuier (10), en el año 2009, realizó un estudio comparativo de la utilización de la escala visual análoga modificada para ansiedad con la State-Trait Anxiety Inventory for Children [STAIC] y la Yale Preoperative anxiety scale modified en el perioperatorio para medir ansiedad en niños de 7 a 16 años, llegando a la conclusión de la utilidad y validez de la escala visual análoga modificada para ansiedad (11).

Uno de los instrumentos de medida que vamos a utilizar para poder valorar el nivel de ansiedad en el paciente es la escala visual análoga modificada utilizada en el estudio de Sepúlveda-Vildósola [2014] sobre la utilización de la musicoterapia para disminuir la ansiedad en pacientes pediátricos sometidos a tratamiento con quimioterapia intravenosa ambulatoria [2].

El dolor crónico desencadena frecuentemente un cuadro de ansiedad, generando un deterioro del bienestar subjetivo en los pacientes. Cuando los pacientes precisan de alguna técnica intervencionista y acuden a la Unidad del Dolor, apreciamos ansiedad situacional.

Desde el entorno hospitalario es fundamental realizar medidas terapéuticas para mejorar la comodidad y la seguridad de los pacientes. Dado que en la actualidad no hay estudios sobre la efectividad de la musicoterapia para reducir la ansiedad ante una técnica intervencionista en la Unidad del Dolor, consideramos que la realización de este estudio está justificada para demostrar que la musicoterapia es una herramienta útil y sencilla, que ayuda a disminuir la ansiedad en las personas que acuden a la Unidad del Dolor para someterse a una técnica intervencionista.

\section{Hipótesis}

La musicoterapia empleada en aquellos pacientes a los que se realiza una técnica intervencionista en la Unidad del Dolor del Hospital Universitario Sagrat Cor de Barcelona disminuye el nivel de ansiedad.

\section{Objetivos}

El objetivo principal es valorar la eficacia de la musicoterapia en la disminución del nivel de ansiedad en aquellos pacientes a los que se les realiza una técnica intervencionista en la Unidad del Dolor del Hospital Universitario Sagrat Cor de Barcelona.

Como objetivos secundarios nos planteamos: valorar la mejoría en la intensidad del dolor según escala visual analógica, cuantificar la variabilidad en los parámetros fisiológicos, valorar si las variables recogidas modifican el grado de ansiedad y determinar la satisfacción de los pacientes tras la realización de la técnica intervencionista con musicoterapia.

\section{MATERIAL Y MÉTODOS}

\section{Diseño del estudio}

Estudio cuasi experimental con grupo control, prospectivo, transversal y no aleatorizado.

\section{Población y muestra}

La población a estudio son los pacientes programados para la realización de una técnica intervencionista en la Unidad del Dolor del Hospital Universitario Sagrat Cor de Barcelona, dos días a la semana, en el turno de mañana, durante tres meses.

En la bibliografía consultada se encuentra una reducción de la ansiedad intensa de un $70 \%$ a un $20 \%$ en pacientes sometidos a odontología tras tratamiento con musicoterapia (11). En este estudio para una prueba unilateral con nivel de confianza del $95 \%$, un poder estadístico del $80 \%$ y una reducción de la ansiedad intensa de un 70 a un $40 \%$, se necesitarían 33 pacientes en cada grupo. Considerando que la pérdida en el seguimiento de estos pacientes durante la intervención puede ser de un máximo del $15 \%$, se necesitarían un total de 39 pacientes en cada grupo.

\section{Criterios de inclusión y exclusión}

Los criterios de inclusión son pacientes mayores de 18 años de edad y que tengan la indicación de la técnica intervencionista (bloqueos, radiofrecuencia). Los criterios de exclusión son pacientes con trastorno psiquiátrico y/o incapaces de contestar las variables del estudio.

\section{Descripción de las variables del estudio}

Las variables que se recogen en el estudio, son las siguientes:

- Variable dependiente: grado de ansiedad: medida según escala visual análoga (Figura 1) en los momentos preintervención, intraintervención y postintervención (técnica intervencionista). Esta escala es una modificación utilizada en un estudio de Sepúlveda-Vildósola [2014].

- Variable independiente: técnica intervencionista a realizar según la indicación y diagnóstico valorado en la visita médica en la Unidad del Dolor del Hospital Universitario Sagrat Cor de Barcelona.

- Variables secundarias: edad (años], sexo del paciente, actividad laboral (recogida según el 


\section{¿Cómo te sientes ahora?}

\section{Considera que 0 es "sin preocupación ni miedo" y 10 es "la peor preocupación o miedo"}

\section{Selecciona conforme los números y las caras lo que mejor describa tu nivel de ansiedad}

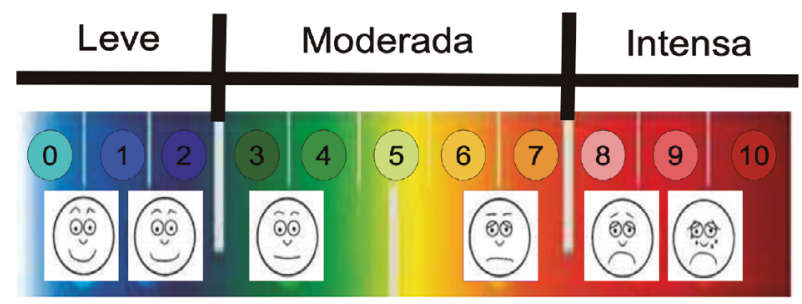

Fig. 1. Escala visual análoga modificada para medir nivel de ansiedad (Sepúlveda-Vildósola, 2014].

paciente se encuentre en activo, de baja, pensionista o desempleado), nivel de estudios (primarios, medios o superiores), diagnóstico y técnica intervencionista a realizar y si esta se realiza por primera vez, tratamiento ansiolítico de manera crónica o puntual, registro de constantes vitales (frecuencia cardiaca en latidos por minutos, saturación de oxígeno por pulsioximetría y tensión arterial no invasiva sistólica y diastólica en milímetros de mercurio) en el preintervención, 2 minutos, 5 minutos, 7 minutos, 10 minutos, 13 minutos, 15 minutos, 20 minutos y postintervención (técnica intervencionista], intensidad del dolor según escala visual analógica [EVA] y grado de satisfacción del paciente según escala de tipo Likert.

\section{Descripción y desarrollo del estudio}

El reclutamiento de los pacientes ha sido el siguiente: los pacientes visitados las tardes de los martes y jueves se han reclutado para el grupo intervención (musicoterapia], la técnica intervencionista se ha realizado el jueves por la mañana. Los pacientes visitados los miércoles y los jueves por la mañana se han reclutado para el grupo control, la técnica intervencionista se ha realizado el martes por la mañana. De esta manera, los pacientes se han reclutado tanto en el grupo intervención (musicoterapia) como en el grupo control durante dos días de visita a la semana y la técnica intervencionista se ha realizado por el facultativo que les ha visitado.

El reclutamiento de los pacientes no ha sido aleatorio, pero el estudio es prospectivo, controlado, y ha seguido la lista de comprobación TREND (Transparent Reporting of Evaluations with Non-Randomized Designs] publicada en el año 2004 (12).

El día de la visita médica en la Unidad del Dolor del Hospital Universitario Sagrat Cor de Barcelona, en la cual se ha programado la técnica intervencionista, el paciente recibe la "Hoja de información para el pacien- te" junto con el "Consentimiento informado" y el "Consentimiento informado de la técnica intervencionista" a realizar; dichos consentimientos los aporta firmados el día de la realización de la técnica intervencionista. La información tanto de la técnica intervencionista como del estudio la realiza el facultativo que posteriormente realizará la técnica intervencionista.

El paciente acude a la Unidad del Dolor del Hospital Universitario Sagrat Cor para la realización de la técnica intervencionista acompañado y con dichos consentimientos firmados.

El personal de enfermería recoge los consentimientos firmados y posteriormente se lleva a cabo el listado de seguridad de verificación para realizar la técnica intervencionista: comprobación de la identidad del paciente, alergias, correcta pauta de retirada $y / 0$ sustitución en caso de tratamiento con antiagregantes y/o anticoagulantes para la realización de la técnica y confirmación de la técnica programada.

El paciente pasa al vestuario, donde se le entrega la ropa pertinente para realizar la técnica intervencionista de manera aséptica.

En los pacientes del grupo intervención (musicoterapia), la sala se ha mantenido con las puertas cerradas y también se han hecho visibles letreros solicitando silencio para crear un espacio libre de contaminación acústica. La música elegida ha sido reproducida mediante el dispositivo electrónico Samsung Galaxy Tab A de forma aleatoria; se ha realizado sobre 184 melodías empleadas en medicina complementaria y alternativa $(13,14)$ una selección de 8 melodías que se han reproducido aleatoriamente; a continuación se describen las mismas: Mantra of Awalokaeshvara: The Meditative Sound of Buddhist Chants, Contemplation: Simply Karma Disc 1, Dreamers: Simply Karma Disc 1, One By One: A Day Without Rain (Enya), Flight Over Africa (John Barry), Beach At Night (John Barry), Over the Horizon: Brand Music y Mozart: Fantasia In $D$ Minor.

Antes de realizar la técnica intervencionista se han registrado los datos demográficos (edad, sexo, actividad laboral, nivel de estudios], si realiza o no tratamiento con ansiolíticos, el diagnóstico y la técnica intervencionista a realizar y si es la primera vez que se realiza la misma.

Se ha evaluado la intensidad del dolor según escala visual analógica (EVA) y la ansiedad según escala visual análoga; simultáneamente se han registrado las constantes vitales (frecuencia cardiaca, tensión arterial no invasiva y saturación de oxígeno por pulsioximetría).

Durante la realización de la técnica intervencionista (intraprocedimiento), se evalúa la intensidad del dolor según EVA y la ansiedad según escala visual análoga, y se registran las constantes vitales (frecuencia cardiaca, tensión arterial no invasiva y saturación de oxígeno por pulsioximetría).

Al finalizar la técnica intervencionista (postprocedimiento), se traslada al paciente a la sala de recuperación para monitorizar posibles efectos adversos y se vuelve a evaluar la intensidad del dolor según EVA y la ansiedad según escala visual análoga; también se registran las constantes vitales (frecuencia cardiaca, tensión arterial no invasiva y saturación de oxígeno por pulsioximetría). 
Al alta del paciente de la Unidad del Dolor del Hospital Universitario Sagrat Cor de Barcelona, se le realiza también un cuestionario de satisfacción.

Durante todo el tiempo en el que ha permanecido el paciente en la Unidad del Dolor, se ha mantenido un ambiente agradable, sin contaminación acústica, y se ha reproducido mediante el dispositivo electrónico Samsung Galaxy Tab A la selección de melodías.

En los pacientes del grupo control, se ha realizado la técnica intervencionista con la misma metodología pero no se les ha aplicado musicoterapia.

\section{Recogida de datos}

De manera prospectiva y transversal, se han recogido por el personal de enfermería las variables descritas anteriormente para su posterior introducción en una base de datos y análisis posterior.

\section{Análisis de los datos}

El periodo de reclutamiento ha sido de tres meses (inclusión del primer caso en junio de 2017, recogida del último caso en febrero de 2018].

Se ha realizado un análisis descriptivo mediante el cálculo de las medias, desviaciones estándar para las variables cuantitativas y el porcentaje para las variables cualitativas.

Para comparar los dos grupos de pacientes, en las variables cualitativas se ha utilizado la prueba de Chi cuadrado o la corrección de Yates, y en las variables cuantitativas la prueba de $\mathrm{T}$ de Student para variables normales o la $U$ de Man-Whitney en el caso de variables de distribución no normal o de varianzas no homogéneas.

Se considera un valor de $p<0,05$ como estadísticamente significativo.

En el tratamiento estadístico de datos se ha utilizado la aplicación estadística Statistics Package Social Sciences (SPSS) versión 19.0.

\section{Aspectos éticos}

Este estudio ha sido revisado y aprobado por el Comité Ético Local de Investigación Clínica del Hospital Universitario Sagrat Cor de Barcelona [2017/13-UDHUSC).

Todos los documentos del estudio serán custodiados en el archivo del hospital por un periodo de diez años, posteriores a su publicación.

El proyecto se ha realizado siguiendo las directrices señaladas por las Buenas Prácticas Clínicas en Investigación y con la Declaración de Helsinki y revisiones sucesivas (versión actualizada en la 64 ${ }^{\mathrm{a}}$ Asamblea General, Fortaleza, Brasil, octubre 2013).

En cumplimento de la Ley Orgánica 15/1999, de 13 de diciembre, de "Protección de Datos de Carácter Personal", la identidad de los pacientes no podrá ser desvelada ni divulgada. Los datos de los pacientes recogidos durante el estudio se documentan de manera anónima y disociada, vinculándose a un código (número de centro y paciente).

\section{RESULTADOS}

Se han reclutado en el estudio un total de 80 sujetos. El 71,6\% han sido mujeres; la edad media de los sujetos ha sido de 66,7 (SD 14) años, el 49,4\% tiene un nivel estudios medios y el $64,2 \%$ son pensionistas.

El 60,5\% de los sujetos no realiza tratamiento con ansiolíticos.

El $59 \%$ de los participantes del estudio presenta lumborradiculalgia y se les ha realizado bloqueo epidural.

En un 33,3\% de los sujetos se les ha realizado la técnica intervencionista por primera vez.

Al comparar el sexo, edad, actividad laboral, nivel de estudios, diagnóstico y técnica intervencionista, tratamiento previo con ansiolíticos, realización de la técnica por primera vez, constantes (frecuencia cardiaca, saturación de oxígeno y tensión arterial), valoración del dolor según escala EVA y valoración de la ansiedad según escala visual análoga antes de la técnica intervencionista, se obtuvo que no había ninguna diferencia estadísticamente significativa entre los pacientes del grupo control y a los que se les realizó la intervención de musicoterapia. Es decir, que los grupos son homogéneos y por lo tanto comparables (Tabla I).

En el grupo control el valor de EVA intraintervención es de 5,83 [SD 3,2) y en el grupo de musicoterapia es inferior, con un valor de EVA de 5,0 (SD 2,2). Esta diferencia no es estadísticamente significativa $(F=1,614$, $p=0,208$ ).

En el grupo control el valor de la escala visual análoga (ansiedad) intraintervención es de 4,3 (SD 3,1) y en el grupo de musicoterapia es inferior, con un valor de 3,0 (SD 2,0). Esta diferencia sí es estadísticamente significativa ( $\mathrm{F}=4,83, p=0,031$ ) (Figura 2).

En el valor de EVA postintervención sucede lo mismo. En el grupo control el valor de EVA postintervención es de 3,7 [SD 3,3) y en el grupo de musicoterapia es inferior, con un valor de EVA de 3,1 [SD 2,4]. Esta diferencia no es estadísticamente significativa $(F=0,755$, $p=0,387$ ).

En el grupo control el valor de la escala visual análoga (ansiedad) postintervención es de 2,7 (SD 2,8) y en el grupo de musicoterapia es inferior, con un valor de 1,3 (SD 1,5). Esta diferencia también es estadísticamente significativa ( $\mathrm{F}=7,427, p=0,008$ ) (Figura 3).

El 81,5\% del total de sujetos que ha participado en el estudio considera que está muy satisfecho y el 18,5\% está satisfecho respecto a haber recibido suficiente información sobre la técnica que se le ha realizado.

El 95,1\% del total de sujetos que han participado en el estudio considera que está muy satisfecho y el 4,9\% está satisfecho, ya que los profesionales sanitarios les han aportado confianza y seguridad.

Los sujetos del grupo intervención refieren que con el empleo de la musicoterapia se ha creado un ambiente relajado y en un $80 \%$ se consideran muy satisfechos y en el $20 \%$ restante se consideran satisfechos.

\section{DISCUSIÓN}

Debido a que los pacientes que acuden a la Unidad del Dolor frecuentemente presentan ansiedad, la finalidad de 
TABLA I

VARIABLES RECOGIDAS PREINTERVENCIÓN

\begin{tabular}{|c|c|c|c|c|}
\hline $\begin{array}{l}\text { Variables estudio } \\
\text { preintervención }\end{array}$ & $\begin{array}{l}\text { Grupo } \\
\text { control }\end{array}$ & $\begin{array}{c}\text { Grupo } \\
\text { musicoterapia } \\
\end{array}$ & Total & $\begin{array}{c}\text { Significación } \\
\text { estadística }\end{array}$ \\
\hline & $\%$ & $\%$ & $\%$ & $\mathrm{p}$ \\
\hline \multicolumn{5}{|l|}{ Sexo } \\
\hline Hombre & 26,8 & 30 & 28,4 & 0,472 \\
\hline Mujer & 73,2 & 70 & 71,6 & \\
\hline \multicolumn{5}{|l|}{ Actividad laboral } \\
\hline Activo & 7,3 & 30 & 18,5 & 0,052 \\
\hline Baja & 14,6 & 12,5 & 13,6 & \\
\hline Desempleado & 2,4 & 5 & 3,7 & \\
\hline Pensionista & 75,6 & 52,5 & 64,2 & \\
\hline \multicolumn{5}{|l|}{ Estudios } \\
\hline Primarios & 41,5 & 30 & 35,8 & 0,336 \\
\hline Medios & 48,8 & 50 & 49,4 & \\
\hline Superiores & 9,8 & 20 & 14,8 & \\
\hline \multicolumn{5}{|l|}{ Diagnóstico } \\
\hline Cefalea cervicogénica & 0 & 2,5 & 1,2 & 0,564 \\
\hline Coxalgia & 0 & 2,5 & 1,2 & \\
\hline Gonalgia & 9,8 & 15 & 12,3 & \\
\hline Lumborradiculalgia & 61 & 55 & 58 & \\
\hline Omalgia & 7,3 & 7,5 & 7,4 & \\
\hline Síndrome facetario & 17,2 & 7,5 & 12,3 & \\
\hline Síndrome miofascial & 4,9 & 10 & 7,4 & \\
\hline \multicolumn{5}{|l|}{ Técnica intervencionista } \\
\hline Bloqueo epidural & 61 & 55 & 58 & 0,306 \\
\hline Bloqueo facetario & 17,1 & 7,5 & 12,3 & \\
\hline Bloqueo interfascial & 4,9 & 10 & 7,4 & \\
\hline Bloqueo nervio occipital mayor & 0 & 2,5 & 1,2 & \\
\hline Bloqueo nervio supraescapular & 7,3 & 2,5 & 4,9 & \\
\hline Bloqueo punto gatillo & 0 & 5 & 2,5 & \\
\hline Radiofrecuencia cadera & 0 & 2,5 & 1,2 & \\
\hline Radiofrecuencia geniculares & 9,8 & 10 & 9,9 & \\
\hline Radiofrecuencia supraescapular & 0 & 5 & 2,5 & \\
\hline \multicolumn{5}{|l|}{ Tratamiento con ansiolíticos } \\
\hline No & 56,1 & 65 & 60,5 & 0,124 \\
\hline Sí, crónico & 34,1 & 35 & 34,6 & \\
\hline Sí, puntual & 9,8 & 0 & 4,9 & \\
\hline \multicolumn{5}{|c|}{ Realización de la técnica por primera vez } \\
\hline Sí & 34,1 & 32,5 & 33,3 & 0,531 \\
\hline No & 65,9 & 67,5 & 66,7 & \\
\hline
\end{tabular}


TABLA I [CONT.]

VARIABLES RECOGIDAS PREINTERVENCIÓN

\begin{tabular}{|c|c|c|c|c|}
\hline $\begin{array}{l}\text { Variables estudio } \\
\text { preintervención }\end{array}$ & $\begin{array}{l}\text { Grupo } \\
\text { control }\end{array}$ & $\begin{array}{c}\text { Grupo } \\
\text { musicoterapia }\end{array}$ & Total & \begin{tabular}{|c} 
Significación \\
estadistica
\end{tabular} \\
\hline & $\%$ & $\%$ & $\%$ & $p$ \\
\hline & Media [SD] & Media [SD] & Media [SD] & $P$ \\
\hline Edad (años) & $69,1(14,1)$ & $64,3(13,7)$ & $66,7(14)$ & 0,123 \\
\hline EVA (dolor) preintervención & $5,83(2,6)$ & $5,8[2,4]$ & $5,8(2,4)$ & 0,958 \\
\hline Escala Visual Análoga (ansiedad) preintervención & $4,1[3,3)$ & $4(2,2)$ & $4(2,8)$ & 0,910 \\
\hline Frecuencia cardiaca (lpm) & $79,7(11,9)$ & $76,4(11,8)$ & $78,1(11,9)$ & 0,222 \\
\hline Saturación oxígeno [\%] & $97(2)$ & $97,3(1,9)$ & $97,2(2)$ & 0,503 \\
\hline Tensión arterial $(\mathrm{mmHg})$ & $141,3[25,3]$ & $142[20,1)$ & $141,6(22,8)$ & 0,89 \\
\hline
\end{tabular}

\% de EVA - INTRA TÉCNICA INTERVENCIONISTA

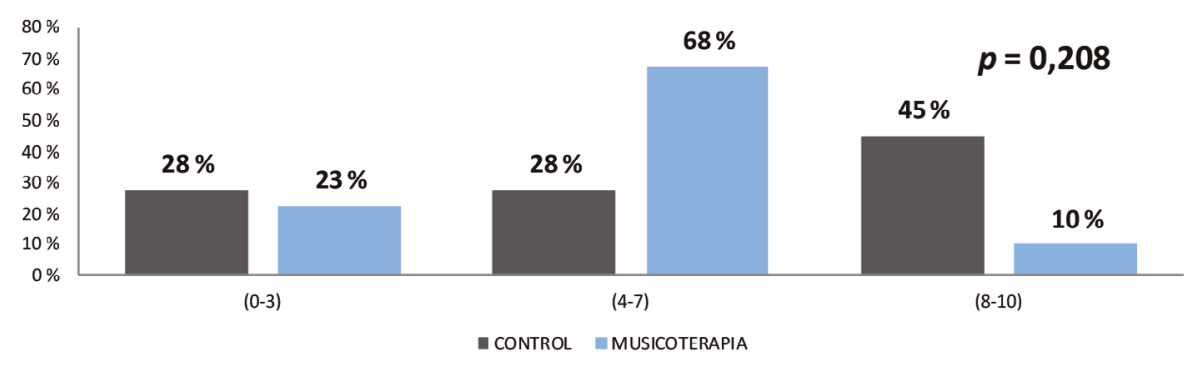

\section{\% de ESCALA VISUAL ANÁLOGA ANSIEDAD - INTRA TÉCNICA INTERVENCIONISTA}

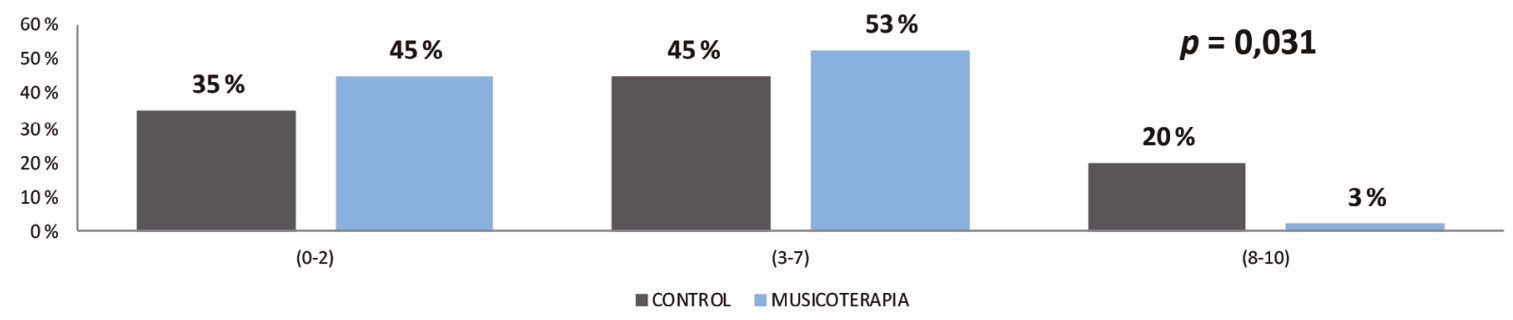

Fig. 2. Resultados intraintervención.

este estudio ha sido demostrar la importancia de reducir el nivel de ansiedad ante la realización de una técnica intervencionista en la Unidad del Dolor.

La musicoterapia consiste en una interacción entre el profesional, el paciente, la música y el medio utilizado para ofrecerla [2]. Tiene como fin obtener beneficios fisiológicos, sociales, psicológicos e intelectuales de los pacientes a los que se les aplica, disminuyendo la ansiedad que está asociada a un estado de estrés negativo, y aumentando el bienestar (15).

Un ambiente tranquilo y confortable, sin contaminación acústica, junto con una colocación adecuada del paciente en la camilla de procedimientos son puntos clave de la asistencia durante la realización de una técnica intervencionista.

La International Association for the Study of Pain (IASP) define el dolor como "una experiencia angustiante asociada con daño tisular real o potencial con componentes sensoriales, emocionales, cognitivos y sociales" (16); precisamente es en el componente emocional sobre el que hemos aplicado la musicoterapia para reducir la ansiedad ante la realización de una técnica intervencionista [17).

Hasta la actualidad, los estudios publicados (18-25) referentes a la ansiedad, el dolor y la musicoterapia han sido realizados en grupos de pacientes sometidos a endoscopias, cistoscopias y colposcopias, biopsias (mama, próstata), curas de pacientes quemados, pacientes trasplantados, mujeres en trabajo de parto y en el postparto, cirugías (cadera, rodilla, cardiaca) y su postoperatorio, pacientes oncológicos y en cuidados paliativos, pacientes sometidos a litotricia extracorpórea con ondas de choque, pacientes con esclerosis múltiple, pacientes sometidos a procedimientos 


\section{\% de EVA - POST TÉCNICA INTERVENCIONISTA}

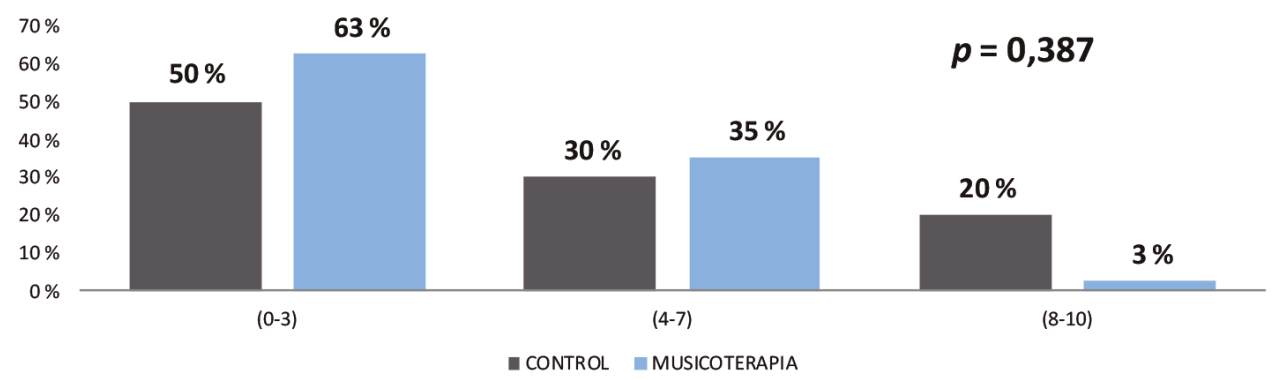

\section{\% de ESCALA VISUAL ANÁLOGA ANSIEDAD - POST TÉCNICA INTERVENCIONISTA}

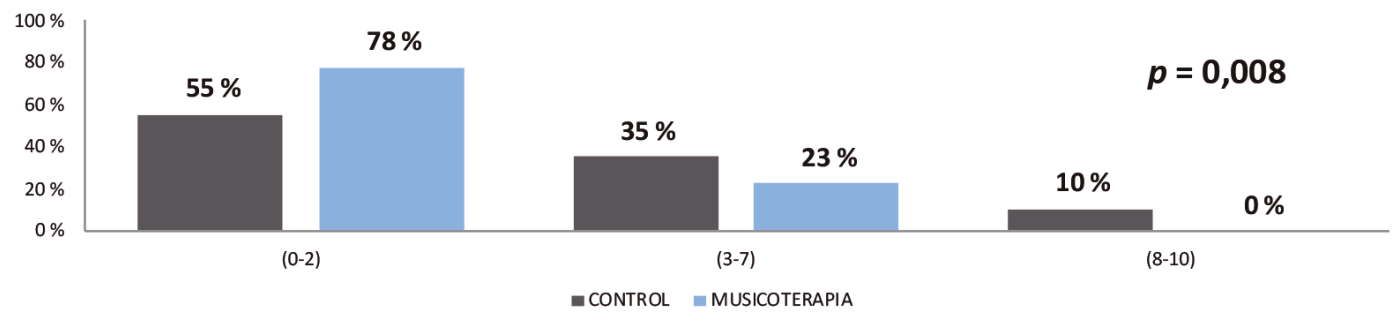

Fig. 3. Resultados postintervención.

radiológicos (nefrostomías, cateterismos) u otro tipo de procedimientos [estética, oftalmológicos, dentales, pleurales). En todos ellos se ha objetivado una disminución en los niveles de ansiedad y en la intensidad del dolor gracias a la musicoterapia, bien por disminución en los requerimientos de fármacos ansiolíticos y/o analgésicos.

Decidimos utilizar como instrumento de medida del nivel de ansiedad la escala visual análoga modificada de Sepúlveda-Vildósola [2] por varias razones: es una escala que se utilizó para valorar el nivel de ansiedad ante la realización de una técnica potencialmente estresante y dolorosa; es un instrumento fácilmente comprensible y de rápida cumplimentación; y se ajusta a los tiempos empleados en la realización de una técnica intervencionista en la Unidad del Dolor del Hospital Universitario Sagrat Cor de Barcelona.

Los resultados obtenidos son similares a los trabajos de Bringuier (10) y Sepúlveda-Vildósola (2) respecto al nivel de ansiedad. Dichos autores estudiaron el nivel de ansiedad en población pediátrica (7-16 años] que presentaba dolor postoperatorio (10) o que se le administraba quimioterapia intravenosa [2]; ambas situaciones, obviamente dolorosas, generan un nivel de ansiedad pre, intra y post.

Nosotros, consideramos extrapolable a la población adulta ambos estudios, ya que en el rango de edad [7-16 años], los sujetos comprenden y manifiestan sus niveles de ansiedad, siendo prácticamente comparables a los pacientes que acuden a la Unidad del Dolor del Hospital Universitario Sagrat Cor de Barcelona para la realización de una técnica intervencionista.
En nuestro estudio, mediante el empleo de musicoterapia, hemos obtenido tanto intraintervención como postintervención (técnica intervencionista), una mejoría clínicamente relevante en la intensidad del dolor medida mediante la escala visual analógica (EVA) y una disminución significativa medida mediante escala visual análoga en la ansiedad tanto intraintervención como postintervención (técnica intervencionista) en el grupo de musicoterapia.

Igualmente, los pacientes se sienten muy satisfechos sobre la información recibida y consideran como muy satisfactorio la confianza y seguridad que les aportan los profesionales sanitarios. Además, el grupo de musicoterapia considera en general muy satisfactorio el ambiente relajado que les crea la música.

Por tanto, tras la valoración de los resultados obtenidos en este estudio, consideramos que la musicoterapia es una excelente herramienta terapéutica, fácil de usar, accesible y económica, con nulos efectos adversos, que puede utilizarse como coadyuvante en las técnicas intervencionistas en la Unidad del Dolor.

No obstante, no podemos atribuir el éxito de estos resultados exclusivamente a la musicoterapia. Hay otros factores externos que han podido influir y que debemos tener en cuenta (ambiente confortable, espacio libre de contaminación acústica, conocimiento de los profesionales sanitarios y espacio donde se realiza la técnica intervencionista].

Como línea de futuro, en función de los resultados obtenidos, consideramos aplicar la musicoterapia como parte integral en la realización de las técnicas intervencionistas en la Unidad del Dolor del Hospital Universitario Sagrat Cor de Barcelona. 


\section{CONFLICTO DE INTERESES}

Los autores declaran no tener ningún conflicto de intereses.

\section{BIBLIOGRAFÍA}

1. NANDA taxonomía etiquetas. Disponible en: https://enfermeriaactual.com/nanda-taxonomia-etiquetas/

2. Sepúlveda-Vildósola AC, Herrera-Zaragoza OR, Jaramillo-Villanueva L, Anaya-Segura A. La musicoterapia para disminuir la ansiedad. Su empleo en pacientes pediátricos con cáncer. Rev Med Inst Mex Seguro Soc. 2014;52(2):50-4.

3. Iriarte Roteta A. Efectividad de la musicoterapia para promover la relajación en pacientes sometidos a ventilación mecánica. Enfermería intensiva. 2003;14(2):43-8. DOI: 10.1016/ S1130-2399(03)78103-6.

4. Franzoi MA, Goulart CB, Lara EO, Martins G. Music listening for anxiety relief in children in the preoperative period: a randomized clinical trial. Rev Lat Am Enfermagem. 2016;24:e2841. DOl: 10.1590/1518-8345.1121. 2841.

5. Sánchez-Camacho P. Musicoterapia: culto al cuerpo y la mente. Envejecimiento activo, envejecimiento en positivo. Logroño: Universidad de la Rioja; 2006. p. 155-88.

6. Ortega E, Esteban L, Estévez AF, Alonso D. Aplicaciones de la musicoterapia en educación especial y en los hospitales. Eur J Educ Psychol. 2009;2(2). DOI: 10.30552/ejep.v2i2.22.

7. Clancy C, Martí P, Mercadal M. La musicoterapia en el contexto quirúrgico. En: 6. ${ }^{\circ}$ Congreso Virtual Internacional de Psiquiatría, Psicología y Enfermería en Salud Mental. Interpsiquis; 2005. 1-5. Disponible en: http://psiqu.com/1-2686.

8. Alonso-Cardaño A, Hernaez-Martínez M, Marti-Auge P. Tratamiento multidisciplinar para el dolor lumbar crónico: Programa de musicoterapia. Rev Soc Esp Dolor. 2008;15(4):22833.

9. Plata-Muñoz, MA, Castillo-Olivares, MA, Guevara-López, UM. Evaluación de afrontamiento, depresión, ansiedad e incapacidad funcional en pacientes con dolor crónico. Rev Mex Anest. 2004;27(1): 16-23.

10. Bringuier S, Dadure C, Raux O, Dubois A, Picot MC, Capdevila $X$. The perioperative validity of the visual analog anxiety scale in children: a discriminant and useful instrument in routine clinical practice to optimize postoperative pain management. Anesth Analg. 2009;109(3):737-44. DOI: 10.1213/ ane.0b013e3181afOOe4.

11. Gómez-Scarpetta RA, Durán-Arismendy L, Cabra-Sosa LJ, Pinzón-Vargas CT, Rodríguez-Becerra NR. Musicoterapia para el control de ansiedad odontológica en niños con síndrome de Down. Revista hacia la Promoción de la Salud. 2012;17(2):13-24.

12. Des Jarlais DC, Lyles C, Crepaz N, TREND Group. Improving the reporting quality of nonrandomized evaluations of beha- vioral and public health interventions: the TREND statement. Am J Public Health. 2004;94(3):361-6. DOI: 10.2105/ ajph.94.3.361.

13. World Health Organization. Legal status of traditional medicine and complementary/alternative medicine: a worldwide review. Geneve WHO, 2001. Disponible en: https://apps. who.int/medicinedocs/pdf/h2943e/h2943e.pdf

14. National Center for Complementary and Alternative Medicine (NCCAM). What is Complementary and Alternative Medicine [internet]. Disponible en: http://nccam.nih.gov.

15. De la Rubia Ortí JE, Sancho Espinós P, Cabañés Iranzo C. Impacto fisiológico de la musicoterapia en la depresión, ansiedad, y bienestar del paciente con demencia tipo Alzheimer. Valoración de la utilización de cuestionarios para cuantificarlo. Eur J investig health psychol educa. 2014;4(2):131-40. DOI: 10.30552/ejihpe.v4i2.69.

16. Williams AC, Craig KD. Pain. Updating the definition of pain Pain. Pain, 2016; Nov 157 (11): 2420-2423. DOI: 10.1097/j.pain.0000000000000613.

17. Orjuela-Rojas JM. Efecto ansiolítico de la musicoterapia: aspectos neurobiológicos y cognoscitivos del procesamiento musical. Rev. Colomb Psiquiat. 2011;40(4):748-59. DOI: 10.1016/S0034-7450(14)60162-1.

18. Xiao Y, Li L, Xie Y, Xu J, Liu Y. Effects of aroma therapy and music intervention on pain and anxious for breast cancer patients in the perioperative period. Zhong Nan Da Xue Xue Bao Yi Xue Ban. 2018;43(6):656-61. DOI: 10.11817/j. issn.1672-7347.2018.06.013.

19. Choi S, Park SG, Lee HH. The analgesic effect of music on cold pressor pain responses: The influence of anxiety and attitude toward pain. PLoS One. 2018;13(8):e0201897. DOI: 10.1371/journal.pone.0201897.

20. Wright J, Adams D, Vohra S. Complementary, holistic, and integrative medicine: music for procedural pain. Pediatr Rev. 2013;34(11):e42-6. DOI: 10.1542/pir.34-11-e42.

21. Kulkarni S, Johnson PC, Kettles S, Kasthuri RS. Music during interventional radiological procedures, effect on sedation, pain and anxiety: a randomised controlled trial. Br J Radiol. 2012;85(1016):1059-63. DOI: 10.1259/ brj/71897605.

22. Nguyen TN, Nilsson S, Hellström AL, Bengtson A. Music therapy to reduce pain and anxiety in children with cancer undergoing lumbar puncture: a randomized clinical trial. J Pediatr Oncol Nurs. 2010;27(3):146-55. DOI: 10.1177/1043454209355983.

23. Nilsson $\cup$. The anxiety- and pain-reducing effects of music interventions: a systematic review. AORN J. 2008;87(4):780807. DOI: 10.1016/j.aorn.2007.09.013.

24. Hoareau SG, De Diego E, Guétin S. Pain management and music therapy. Rev Infirm. 2016;(217):31-3. DOI: 10.1016/revinf.2015.10.025.

25. Yinger OS, Gooding LF. A systematic review of musicbased interventions for procedural support. J Music Ther. 2015;52(1):1-77. DOI: 10.1093/jmt/thv004. 